\title{
A New Look at the Final Period Decay of
}

\section{Homogeneous Isotropic Turbulence}

\author{
C. Mamaloukas \\ Department of Statistics \\ Athens University of Economics and Business, Greece \\ mamkris@aueb.gr
}

Copyright (c) 2013 C. Mamaloukas. This is an open access article distributed under the Creative Commons Attribution License, which permits unrestricted use, distribution, and reproduction in any medium, provided the original work is properly cited.

\begin{abstract}
Final-period decay law in homogeneous and isotropic turbulence is reexamined here from the concept of a simple point-merging technique.
\end{abstract}

Keywords: Homogeneous and isotropic turbulence, simple point-merging technique

\section{Introduction}

Ghosh (1972) investigated the early-period decay process of a general type of turbulence using quasi-normality hypothesis. He established two lemmas concerning merger of points in the physical space.

\section{First lemma:}

This lemma is concerned with the behavior of correlation tensors in the energy space when two or more points under reference coincide.

Let $\Psi_{i, j}\left(\vec{\kappa}, \overrightarrow{\kappa^{\prime}}, t\right)$ and $\Psi_{i, j, \kappa}\left(\vec{\kappa}, \overrightarrow{\kappa^{\prime}}, \overrightarrow{\kappa^{\prime \prime}}, t\right)$ are spectrum functions which correspond respectively to correlation function $F_{i, j}\left(x, x^{\prime}, t\right)\left[=\overline{u_{i} u_{j}^{\prime}}\right]$ and $F_{i, j, \kappa}\left(x, x^{\prime}, x^{\prime \prime}, t\right)\left[=\overline{u_{i} u_{j}^{\prime} u_{\kappa}^{\prime \prime}}\right]$, pertaining to points, say $x, x^{\prime}, x^{\prime \prime}$ at the same instant of time $t$ in the turbulent medium. When the third point $x^{\prime \prime}$ merges with the first point $\mathrm{x}$, then we have: 


$$
\int \Psi_{i, j, \kappa}\left(\overrightarrow{\lambda-\kappa^{\prime \prime}}, \overrightarrow{\kappa^{\prime}}, \overrightarrow{\kappa^{\prime \prime}}, t\right) d \overrightarrow{\kappa^{\prime \prime}}=\Psi_{i k, j}\left(\lambda, \overrightarrow{\kappa^{\prime}}, t\right)
$$

where $\vec{\lambda}=\vec{\kappa}+\overrightarrow{\kappa^{\prime}}$

\section{Second lemma:}

The second lemma is concerned with the Millionschikov's quasi-normality hypothesis. We consider additional fluctuation velocity component $u_{l}^{\prime \prime \prime}$ at the point $x^{\prime \prime \prime}$, and the spectrum tensor $\Psi_{i, j, \kappa, l}\left(\vec{\kappa}, \overrightarrow{\kappa^{\prime}}, \overrightarrow{\kappa^{\prime \prime}}, \overrightarrow{\kappa^{\prime \prime \prime}}, t\right)$ which correspond to correlation function $F_{i, j, \kappa, l}\left(x, x^{\prime}, x^{\prime \prime}, x^{\prime \prime \prime}, t\right)\left[=\overline{u_{i} u_{j}^{\prime} u_{\kappa}^{\prime \prime} u_{l}^{\prime \prime \prime}}\right]$.

When the fourth point $x^{\prime \prime \prime}$ coincides with the first point $x$, we derive the relation

$$
\begin{aligned}
\Psi_{i l, j, \kappa}\left(\vec{\kappa}, \overrightarrow{\kappa^{\prime}}, \overrightarrow{\kappa^{\prime \prime}}, t\right) & =\int \Psi_{i, j}\left(\overrightarrow{\kappa-\kappa^{\prime \prime \prime}}, \overrightarrow{\kappa^{\prime}}, t\right) \Psi_{\kappa, l}\left(\overrightarrow{\kappa^{\prime \prime}}, \overrightarrow{\kappa^{\prime \prime \prime}}, t\right) d \overrightarrow{\kappa^{\prime \prime \prime}} \\
& +\int \Psi_{i, j}\left(\overrightarrow{\kappa-\kappa^{\prime \prime \prime}}, \overrightarrow{\kappa^{\prime \prime}}, t\right) \Psi_{j, l}\left(\overrightarrow{\kappa^{\prime}}, \overrightarrow{\kappa^{\prime \prime \prime}}, t\right) d \overrightarrow{\kappa^{\prime \prime \prime}} \\
& +\int \Psi_{i l}(\vec{\kappa}, t) \Psi_{j, \kappa}\left(\overrightarrow{\kappa^{\prime}}, \overrightarrow{\kappa^{\prime \prime}}, t\right)
\end{aligned}
$$

If the third point $x^{\prime \prime}$ merges with the second point $x^{\prime}$, we derive the relation,

$$
\begin{aligned}
& \Psi_{i l, j \kappa}\left(\vec{\kappa}, \overrightarrow{\kappa^{\prime}}, t\right)=\iint\left[\Psi_{i, j}\left(\overrightarrow{\kappa-\kappa^{\prime \prime \prime}}, \overrightarrow{\kappa^{\prime}-\kappa^{\prime \prime}}, t\right) \Psi_{\kappa, l}\left(\overrightarrow{\kappa^{\prime \prime}}, \overrightarrow{\kappa^{\prime \prime \prime}}, t\right)\right. \\
& \left.+\Psi_{i, \kappa}\left(\overrightarrow{\kappa-\kappa^{\prime \prime \prime}}, \overrightarrow{\kappa^{\prime \prime}}, t\right) \Psi_{j, l}\left(\overrightarrow{\kappa^{\prime}-\kappa^{\prime \prime}}, \overrightarrow{\kappa^{\prime \prime \prime}}, t\right)\right] d \overrightarrow{\kappa^{\prime \prime}} d \overrightarrow{\kappa^{\prime \prime \prime}} \\
& +\Psi_{i l}(\vec{\kappa}, t) \Psi_{j, \kappa}\left(\overrightarrow{\kappa^{\prime}}, t\right)
\end{aligned}
$$

The relation (1.3) is derived from Millionschikov's quasi-normality hypothesis e.g.,

$$
\overline{u_{i} u_{j}^{\prime} u_{k}^{\prime \prime} u_{l}^{\prime \prime \prime}}=\overline{u_{i} u_{j}^{\prime}} \overline{u_{k}^{\prime \prime} u_{l}^{\prime \prime \prime}}+\overline{u_{i} u_{k}^{\prime \prime}} \overline{u_{j}^{\prime} u_{l}^{\prime \prime \prime}}+\overline{u_{i} u_{l}^{\prime \prime \prime}} \overline{u_{j}^{\prime} u_{k}^{\prime \prime}}
$$

Ghosh (1972) derived the early period decay equation e.g.,

$$
\frac{\partial^{2}}{\partial t^{2}} \Psi_{i, j}\left(\vec{\kappa}, \overrightarrow{\kappa^{\prime}}, t\right)=I_{1}\left(\vec{\kappa}, \overrightarrow{\kappa^{\prime}}, t\right)+I_{2}\left(\vec{\kappa}, \overrightarrow{\kappa^{\prime}}, t\right)
$$

for the general type of turbulence, where $I_{1}\left(\kappa, \kappa^{\prime}, t\right)$ and $I_{2}\left(\kappa, \kappa^{\prime}, t\right)$ have the requisite expressions (see the original paper of Ghosh (1972)). Further, Ghosh showed that for homogeneous and isotropic turbulence the equation (1.5) reduces to:

$$
\frac{\partial^{2}}{\partial t^{2}} \Psi_{i, j}\left(\vec{\kappa}, \overrightarrow{\kappa^{\prime}}, t\right)=I_{1}\left(\vec{\kappa}, \overrightarrow{\kappa^{\prime}}, t\right)
$$

Expressing the second rank spectrum tensor for homogeneous and isotropic turbulence $\Psi_{m, n}(\vec{\kappa}, \overrightarrow{-\kappa}, t)$, as 


$$
\Psi_{m, n}(\vec{\kappa}, \overrightarrow{-\kappa}, t)=\frac{F(\kappa, t)}{4 \pi \kappa^{2}}\left\{\delta_{m n}-\frac{\boldsymbol{\kappa}_{m} \boldsymbol{\kappa}_{n}}{\boldsymbol{\kappa}^{2}}\right\}
$$

he obtained finally the Proudman-Reid equation e.g.,

$$
\frac{\partial^{2}}{\partial t^{2}} \int_{0}^{\infty} 2 \boldsymbol{\kappa}^{2} F(\boldsymbol{\kappa}, t) d \boldsymbol{\kappa}=\frac{4}{3}\left[\int_{0}^{\infty} \boldsymbol{\kappa}^{2} F(\boldsymbol{\kappa}, t) d \boldsymbol{\kappa}\right]^{2}
$$

In the present note, we however solve the final period decay equation of homogeneous isotropic turbulence using the concept of point-merging technique of Ghosh (1972), as explained above.

\section{Final-period decay equation of homogeneous and isotropic turbulence}

In this case, we would use the Ghosh's lemma (1.1), as applied here to the merging of second point to the first point in a two point correlation tensor, as

$$
\int \Psi_{i, j}\left(\overrightarrow{\kappa-\kappa^{\prime}}, \overrightarrow{\kappa^{\prime}}, t\right) d \overrightarrow{\kappa^{\prime}}=\Psi_{i j}(\vec{\kappa}, t)
$$

The decay equation for the homogeneous and isotropic turbulence may be read as (Hinze, 1959)

$$
\frac{\partial}{\partial t} \Psi_{i, j}\left(\vec{\kappa}, \overrightarrow{\kappa^{\prime}}, t\right)=T_{i, j}\left(\vec{\kappa}, \overrightarrow{\kappa^{\prime}}, t\right)-v\left(\kappa^{2}+\kappa^{\prime 2}\right) \Psi_{i, j}\left(\vec{\kappa}, \overrightarrow{\kappa^{\prime}}, t\right)
$$

where $T_{i, j}\left(\vec{\kappa}, \overrightarrow{\kappa^{\prime}}, t\right)$ represents the non-linear transfer of homogeneous and isotropic turbulence energy (i.e. through a cascade of energy transfer process).

For the final-period decay process, the equation (2.2) reduces to the simple case e.g.,

$$
\frac{\partial}{\partial t} \Psi_{i, j}\left(\vec{\kappa}, \overrightarrow{\kappa^{\prime}}, t\right)=-v\left(\kappa^{2}+\kappa^{\prime 2}\right) \Psi_{i, j}\left(\vec{\kappa}, \overrightarrow{\kappa^{\prime}}, t\right)
$$

Solution of this equation is given by

$$
\Psi_{i, j}\left(\vec{\kappa}, \overrightarrow{\kappa^{\prime}}, t\right)=\Psi_{i, j}\left(\vec{\kappa}, \overrightarrow{\kappa^{\prime}}, t_{0}\right) e^{-v\left(\kappa^{2}+\kappa^{\prime 2}\right) t}
$$

where $t_{0}$ is an initial instant of time.

We express the above equation in the form:

$$
\iint \Psi_{i, j}\left(\overrightarrow{\kappa-\kappa^{\prime}}, \overrightarrow{\kappa^{\prime}}, t\right) d \vec{\kappa} d \overrightarrow{\kappa^{\prime}}=\iint \Psi_{i, j}\left(\overrightarrow{\kappa-\kappa^{\prime}}, \overrightarrow{\kappa^{\prime}}, t_{0}\right) e^{-v\left[\left(\kappa-\kappa^{\prime}\right)^{2}+k^{\prime 2}\right] t} d \vec{\kappa} d \overrightarrow{\kappa^{\prime}}
$$

or

$$
\int \Psi_{i j}(\vec{\kappa}, t) d \vec{\kappa}=\int \Psi_{i j}\left(\vec{\kappa}, t_{0}\right) e^{-v \kappa^{2} t} d \vec{\kappa}
$$


Let us introduce now the transformations:

$$
\begin{aligned}
d \wp=d \kappa_{1} d \kappa_{2} d \kappa_{3} \quad ; \quad & \kappa_{1}=\wp \sin \theta \cos \phi \\
& \kappa_{2}=\wp \sin \theta \sin \phi \\
& \kappa_{3}=\wp \cos \theta
\end{aligned}
$$

where $\wp=|\vec{\kappa}|$

Therefore,

$$
\begin{aligned}
& \int \Psi_{i j}(\wp, t) d \wp=\int_{0}^{\infty} \int_{0}^{2 \pi} \int_{0}^{\pi} \Psi_{i j}\left(\wp, t_{0}\right) e^{-v \wp^{2} t} \wp^{2} \sin \theta d \theta d \phi d \wp= \\
& =2 \cdot 2 \pi \int_{0}^{\infty} \Psi_{i j}\left(\wp, t_{0}\right) e^{-v \wp^{2} t} \wp^{2} d \wp= \\
& =\frac{4 \pi}{2} \int_{0}^{\infty} \Psi_{i j}\left(s, t_{0}\right) s^{\frac{1}{2}} e^{-p s} d s \quad \text { where } v t=p, \wp^{2}=s \\
& \int \Psi_{i i}(\vec{\kappa}, t) d \vec{\kappa}=2 \pi \int_{0}^{\infty} 2 A_{0} \wp^{2} s^{\frac{1}{2}} e^{-p s} d s=4 \pi A_{0} \int_{0}^{\infty} s^{\frac{3}{2}} e^{-p s} d s= \\
& =4 \pi \frac{\Gamma\left(\frac{5}{2}\right)}{p^{\frac{5}{2}}}=4 \pi \frac{3}{4} \sqrt{\pi} \frac{1}{(v t)^{\frac{5}{2}}}=\frac{3 \pi^{\frac{3}{2}}}{(v t)^{\frac{5}{2}}}
\end{aligned}
$$

where $A_{0}$ is constant.

Now,

$$
\frac{1}{2} \int \Psi_{i i}(\vec{\kappa}, t) d \vec{\kappa}=\frac{1}{2} \overline{u_{i}^{2}}, \text { say }
$$

and in isotropic turbulence

$$
\frac{1}{2}\left(\overline{u_{1}^{2}}+\overline{u_{2}^{2}}+\overline{u_{3}^{2}}\right)=\frac{3}{2} u^{\prime 2}=\frac{3 \pi^{\frac{3}{2}}}{(v t)^{\frac{5}{2}}}, \text { say }
$$

Thus,

$$
u^{\prime 2} \approx t^{-\frac{5}{2}}
$$

Hinze (1959) discussed that the inverse five-halves decay law for $u^{\prime 2}$ is obtained on the assumption of analytic behavior at $k=0$ of the energy-spectrum tensor $E_{i, i}$. In his text Davidson (2005) also discussed elaborately that in the case of final-period decay of isotropic turbulence, the non-linear term becomes unimportant and we have the longitudinal correlation function as 


$$
f \approx \exp \left[\frac{-r^{2}}{(8 v t)}\right]
$$

and

$$
u^{\prime 2} \approx t^{-\frac{5}{2}}
$$

\section{Conclusions}

One may conclude that the point-merging technique may be treated as an important concept and applied in deriving relations in homogeneous turbulence.

\section{References}

[1] Davidson P.A., Turbulence: An Introduction for Scientists and Engineers, Oxford University, Oxford, England, (2005).

[2] Ghosh K.M., Some Concequences of Millionschikov's Hypothesis in the Early-Period Decay Process of Turbulence, Indian Journal of Pure and Appl. Math.,Vol. 3, No.1, (1972), 157.

[3] Hinze J.O., Turbulence, McGraw-Hill, New York, (1959).

[4] Mazumdar H.P., On the decay process of turbulence at large Reynolds and Peclet numbers, App. Sci. Res., Vol. 32, (1976), 571.

[5] Mazumdar H.P., On Incompressible Hydromagnetic Turbulence, (Research monograph), Calcutta Mathematical Society, (2010).

[6] Millionschikov M., On the theory of homogeneous isotropic turbulence, Dokl . Akad. Nauk SSSR, 32, (1941), 615-618.

[7] Monin A.S. and Yaglom A.M., Statistical Fluid Mechanics, Vol. II, MIT Press, Cambridge, MA, (1975).

[8] Proudman I., Reid W.H., On the Theory of a Normally Distributed and Homogeneous Turbulent Velocity Field., Phil. Trans. R. Soc. 247A, (1954), 163-189.

Received: November, 2012 